\title{
Physiologic load-bearing characteristics of autografts, allografts, and polymer-based scaffolds in a critical sized segmental defect of long bone: an experimental study
}

\author{
This article was published in the following Dove Press journal: \\ International Journal of Nanomedicine \\ 23 April 2013 \\ Number of times this article has been viewed
}

\section{LF Amorosa' \\ $\mathrm{CH}$ Lee $^{2}$ \\ AB Aydemir ${ }^{1}$ \\ S Nizami' \\ A Hsu' \\ NR Patel' \\ TR Gardner' \\ A Navalgund ${ }^{3}$ \\ D-G Kim ${ }^{3}$ \\ SH Park ${ }^{4}$ \\ JJ $\mathrm{MaO}^{2}$ \\ FY Lee'}

'Center for Orthopaedic Research, Columbia University Medical Center, New York, NY, ${ }^{2}$ College of Dental Medicine, Columbia University Medical Center, New York, NY, ${ }^{3}$ Orthodontics, College of Dentistry, Ohio State University, Columbus, $\mathrm{OH}$ ${ }^{4}$ Department of Orthopaedic Surgery, University of California at Los Angeles Medical Center, Los Angeles, CA, USA
Correspondence: Francis Young-In Lee Center for Orthopaedic Research, Columbia University Medical Center, 630 W 168th St, New York, NY 10032, USA Tel + I 212305 I5I5

Email fl|27@columbia.edu
Background: To address the challenge of treating critical sized intercalary defects, we hypothesized that under physiologic cyclic loading, autografts, allografts, and scaffolds loaded with and without human mesenchymal stem cells (hMSCs) would have different biomechanical characteristics.

Methods: Using a rat femoral defect model, 46 rats were assigned to four groups, ie, autograft $(n=12)$, allograft $(n=10)$, scaffold $(n=13)$, and scaffold with hMSCs $(n=11)$. The scaffold groups used a $5 \mathrm{~mm}$ segment of scaffold composed of $80 \%$ poly- $\varepsilon$-caprolactone and $20 \%$ hydroxyapatite. Rats were sacrificed 4 months postoperatively, and the repairs were assessed radiographically and biomechanically.

Results: Autograft and allograft groups exhibited the most bridging callus, while the scaffold/ hMSCs group had more callus than the scaffold repairs. Although signs of radiographic healing did not accurately reflect restoration of mechanical properties, addition of hMSCs on the scaffold enhanced bone formation. The scaffold alone group had significantly lower elastic and viscous stiffness and higher phase angles than other repairs and the contralateral controls. Addition of hMSCs increased the elastic and viscous stiffness of the repair, while decreasing the phase angle.

Conclusion: Further comparative analysis is needed to optimize clinical use of scaffolds and hMSCs for critical sized defect repairs. However, our results suggest that addition of hMSCs to scaffolds enhances mechanical simulation of native host bone.

Keywords: fracture healing, scaffolds, human mesenchymal stem cells, tissue engineering

\section{Introduction}

Critical sized intercalary defects are commonly encountered in high-energy skeletal traumas or musculoskeletal oncologic reconstructive procedures. Although autogenous bone grafts and vascularized fibular grafts are commonly used for the treatment of large structural defects, they are limited by donor site morbidity, graft size/shape mismatch, and lack of bridging for critical sized defects larger than $6 \mathrm{~cm}$. Structural allografts have been alternatively used for massive segmental bone defects, but suffer from problems of infection, failure of incorporation, decreased mechanical properties over time, and fractures. ${ }^{1,2}$

Recently, there has been a drive to engineer scaffolds that have the ability to incorporate host bone and the capability to provide structural support for large bone defects. With the aid of computer modeling, three-dimensional scaffolds may be 
generated with anatomically accurate bone structures and which match the size and shape of the missing bone. Scaffolds have the theoretical benefit of providing osteoconductivity while potentially serving as carriers for osteoinductive agents and osteoprogenitors, such as mesenchymal stem cells. ${ }^{3,4}$ Although several different polymer-based scaffolds, such as beta-tricalcium phosphate, are currently under investigation as potential models for regenerative tissue engineering, poly- $\varepsilon$-caprolactone (PCL) scaffolds have yielded promising results in prior studies. ${ }^{5-7}$ Most notably, a poly- $\varepsilon$-caprolactone and hydroxyapatite (PCL-HA) scaffold was used successfully to regenerate an osteochondral defect in the proximal humerus of the rabbit. ${ }^{8}$ This scaffold was associated with bone and cartilage regeneration, suggesting that PCL-HA scaffolds may also have inherent homing ability to attract autogenous stem cells. Therefore, we selected this promising polymer scaffold as the basis for our rat femoral segmental defect model, and evaluated the load-bearing capacity of the scaffold repair under cyclic physiologic loading in conjunction with radiographic assessments.

We hypothesized that autografts, allografts, and PCL-HA scaffolds with and without human mesenchymal stem cells (hMSCs) would have different biomechanical load-bearing characteristics under cyclic physiologic loading. Our hypothesis was based on the fact that these grafts have different cellular compositions and physicochemical properties. To test our hypothesis, we used a rat femoral segmental defect model, which has been used previously to test the effects of various materials and agents on bone growth. ${ }^{9-14}$ We created a $5 \mathrm{~mm}$ femoral defect, replaced the defect with appropriate grafts, and repaired the construct using an intramedullary K-wire. Segmental defects of $5 \mathrm{~mm}$ in the rat femurs have been shown to result consistently in nonunions, both radiographically and clinically. ${ }^{15}$ Fixation with an intramedullary K-wire has been used in prior segmental defect studies, and such constructs translate to the most common clinical scenario of intramedullary fixation of the femur and tibia. ${ }^{16,17}$ In our study, rat femoral repairs were evaluated postoperatively with radiographs and noninvasive dynamic mechanical analysis, which simultaneously assessed the elastic and viscoelastic properties of bone under low amplitude physiologic cyclic loading. ${ }^{18-22}$ Our objective was to compare cyclic load-bearing characteristics and radiographic outcomes of each repair type.

\section{Materials and methods In vivo animal experiments}

Institutional Animal Care and Use Committee approval was obtained prior to the start of this study. The study design was based on a rat femoral segmental defect model using an intramedullary K-wire for fixation. ${ }^{12,13} \mathrm{~A}$ total of 46 rats were used to test four different groups. In the autograft group ( $n=12$ ), the mid-diaphysis of the femur was transected at two locations $5 \mathrm{~mm}$ apart, and the original host bone was maintained in the defect. For the allograft group $(n=10)$, a $5 \mathrm{~mm}$ segment of rat femoral diaphysis from another female rat of the same strain was implanted into the defect. The allografts were harvested with their periosteum removed, washed with hydrogen peroxide and sterile saline, frozen at $-80^{\circ} \mathrm{C}$, and then defrosted prior to implantation. In the scaffold alone group $(n=13)$, scaffolds comprised of PCL-HA were implanted into the defects. Lastly, the scaffold/hMSC group used scaffolds loaded with two million hMSCs each $(\mathrm{n}=11)$.

\section{Design and fabrication of scaffold}

The right femur was harvested from a representative weightmatched three-month-old female Sprague-Dawley rat (Charles River Laboratories, Wilmington, MA, USA) for imaging by microcomputed tomography (Stratec XCT-3000, running the XCT Series software, version 5.21; Stratec Medizintechnik, Phorzheim, Germany). Slice thickness was $1 \mathrm{~mm}$, with $0 \mathrm{~mm}$ between cuts. Voxel size was $0.1 \times 0.1 \times 1.0 \mathrm{~mm}$. From the axial cuts, the three-dimensional contour of the femur was reconstructed using 3D Doctor (Able Software Corporation, Lexington, MA, USA) and CAD software (Rhinoceros, McNeel, Seattle, WA, USA, Figure 1). Using these anatomic dimensions, a composite polymer scaffold was fabricated via layer-by-layer

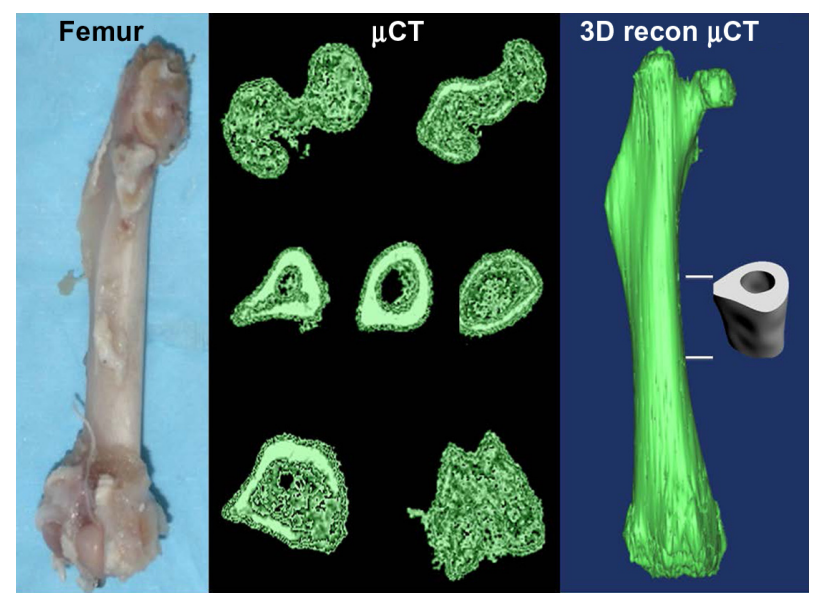

Figure I Scaffold reconstruction.

Notes: Rat femur (left panel) was scanned using microcomputed tomography to obtain two-dimensional cross-sectional areas (middle panel). These axial computed tomography cuts were compiled to generate a three-dimensional femoral reconstruction (right panel). Using the dimensions from this model, a $5 \mathrm{~mm}$ segment of the composite polymer scaffold was fabricated from poly- $\varepsilon$-caprolactone and hydroxyapatite. 
deposition using a three-dimensional printing system (BioplotterTM, EnvisionTec GmbH, Gladbeck, Germany). The composite consisted of $80 \%$ PCL (molecular weight approximately 65,000 , Sigma-Aldrich, St Louis, MO, USA) and 20\% of HA (Sigma-Aldrich) by weight. The composite was melted in the chamber at $120^{\circ} \mathrm{C}$ and dispensed through a 27 -gauge metal needle (DL Technology, Haverhill, MA, USA) to construct a scaffold with interlaid strands and interconnected microchannels (diameter $200 \mu \mathrm{m}$ ). An intramedullary canal with a diameter of $1.8 \mathrm{~mm}$ was created to allow passage of a $1.6 \mathrm{~mm} \mathrm{~K}$-wire. The scaffold was sterilized in ethylene oxide for 24 hours prior to implantation.

\section{Human mesenchymal stem cell implantation}

Human MSCs were obtained from Lonza (Walkersville, MD, USA) and kept frozen in liquid nitrogen. The cells were thawed at passage four and cultured in basal stem cell medium for two days. The scaffolds were prewetted with sterile phosphate-buffered solution and were each seeded with two million hMSCs in a final volume of $40 \mu \mathrm{L} / \mathrm{scaf}$ fold. Cell suspension that leaked out of the scaffold was added back onto the scaffold using a micropipette. After five hours, complete osteogenic medium was added to the wells, and the scaffolds remained in the incubator overnight. The stem cells were tagged with luciferase and mCherry to allow visual verification of cell attachment to the scaffold using a Kodak In-Vivo Multispectral Imaging System FX (Eastman Kodak, Rochester, NY, USA).

\section{Surgery}

Three-month-old female Sprague-Dawley rats (Charles River Laboratories) were used for the autograft, allograft, and scaffold groups, while nude athymic rats were used for the scaffold/hMSC group to minimize immunoreactivity to hMSCs. Isoflurane was inhaled for anesthesia while buprenorphine $0.05 \mathrm{mg} / \mathrm{kg}$ was injected subcutaneously for analgesia. Prior to incision, penicillin G 22,000 IU/kg was injected intramuscularly. The rat was placed in the lateral position, and the right hindquarter was shaved and sterilized with alcohol and povidone-iodine. A longitudinal incision was made lateral to the femur, and the intermuscular septum was divided to expose the femur. At the middle of the diaphysis, the periosteum was stripped from a $5 \mathrm{~mm}$ bone segment, and the segment was cut and removed. Next, a fully threaded $1.6 \mathrm{~mm} \mathrm{~K}$-wire (Zimmer, Warsaw, IN, USA) was drilled in an antegrade fashion though the distal segment of the femur out of the flexed knee joint. Following this, the graft was placed into the defect. The
$\mathrm{K}$-wire was then drilled in a retrograde fashion proximally through the distal femoral segment, the graft, and into the proximal femur through the cortex of the greater trochanter (Figure 2). The wire was cut flush with the surface of the trochlear groove of the knee before closing the wound in layers. Rats were allowed to bear weight immediately. Four months later, the rats were euthanized in a carbon dioxide chamber. Both femurs were harvested, wrapped in saline-soaked gauze, and stored at $-21^{\circ} \mathrm{C}$.

\section{Radiographic assessment}

Anteroposterior and lateral radiographs of the femurs were taken using the Kodak In-Vivo Multispectral Imaging System. Radiographic healing was assessed by three independent and blinded orthopedic surgeons not otherwise involved with the study. Radiographs were graded based on the number of bridging calluses at the proximal and distal cortex-graft interfaces.

\section{Biomechanical testing}

All femurs were thawed and measured prior to testing at room temperature. The head and distal end of the representative femurs from each group were glued to the plate of the testing device, and an axial load was applied to the head of the femur in a proximal to distal direction (Figure 3). The specimens were subjected to dynamic mechanical analysis

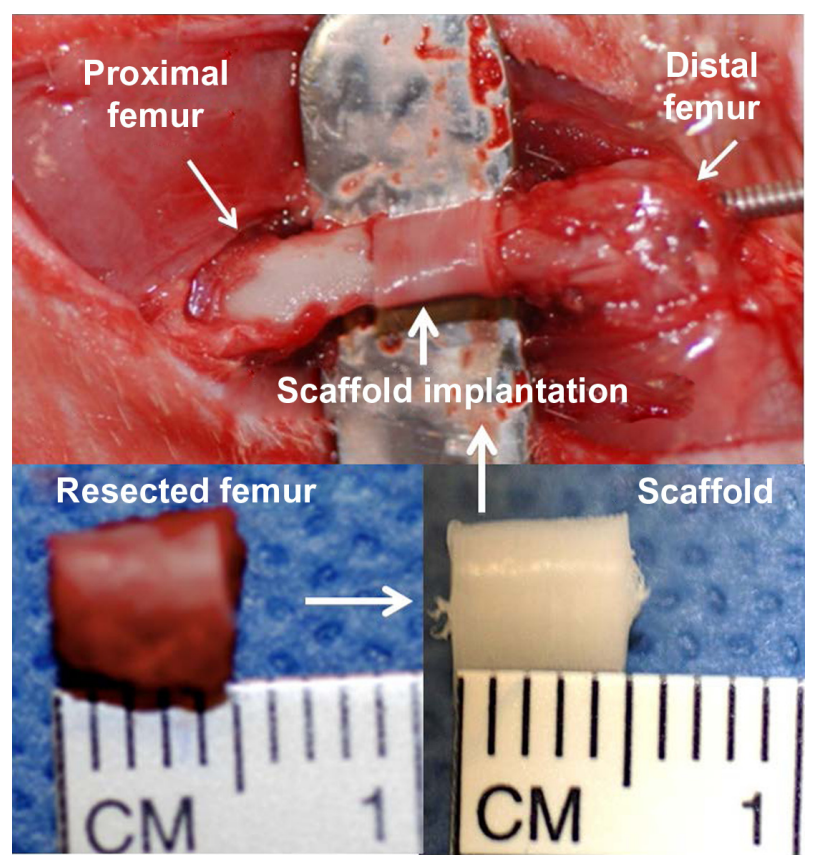

Figure 2 Scaffold placement.

Note: Intraoperative photograph demonstrates a rat femur with a $5 \mathrm{~mm}$ scaffold in place, and the K-wire is noted to exit distally through the trochlear groove of the knee. 

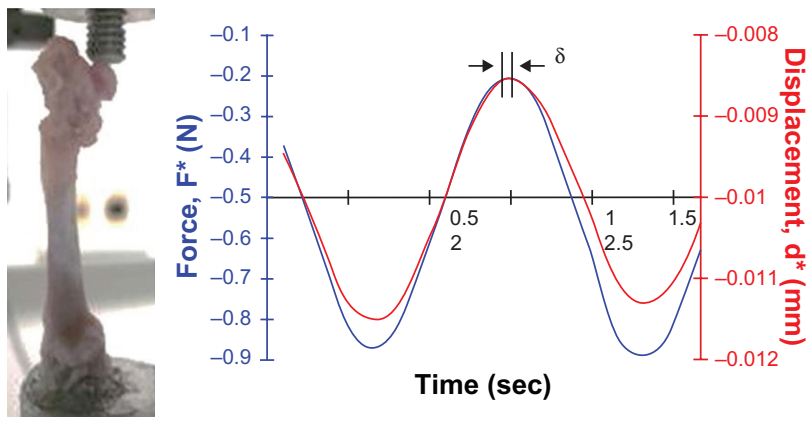

Figure 3 Biomechanical testing.

Note: A high resolution ( $15 \mathrm{~nm}$ ) displacement transducer was used to evaluate the elastic stiffness, viscous stiffness, and phase angle of all femoral constructs.

under nondestructive cyclic axial displacement at $0.5,1.0$, and $5.0 \mathrm{~Hz}$ (Bose Corporation, Framingham, MA, USA). The applied cyclic displacement was estimated based on the specimen height corresponding to the cyclic strain in the range of $20-2020 \mu \varepsilon$, to mimic physiologic loading strains. ${ }^{23}$ A high resolution $(15 \mathrm{~nm})$ displacement transducer was used to control displacement. Dynamic stiffness was measured using dynamic force $\left(\mathrm{F}^{*}\right)$ and displacement $\left(\mathrm{d}^{*}\right)$ (Figure 3). The dynamic (complex) stiffness $\left(\mathrm{K}^{*}\right)$ is composed of two parameters, ie, elastic (storage) stiffness $\left(\mathrm{K}^{\prime}\right)$ and viscous (loss) stiffness $\left(\mathrm{K}^{\prime \prime}\right)$, as defined by the following equation: $\mathrm{K}^{*}=\mathrm{K}^{\prime}+i \mathrm{~K}^{\prime \prime}$, where $\mathrm{K}^{\prime}=\mathrm{K}^{*} \cos (\delta)$ and $\mathrm{K}^{\prime \prime}=\mathrm{K}^{*} \sin (\delta)$.

The elastic and viscous stiffness of bone represent its abilities to store and lose energy in response to the applied load, respectively. ${ }^{24}$ The phase angle is the inverse tangent of the ratio of the loss stiffness to the storage stiffness, and is expressed in degrees. As such, the phase angle accounts for the efficiency of bone in dissipation of energy independent of any structural variation of the femur. The greater the phase angle, the more energy is dissipated.

\section{Statistical analysis}

The SAS program (SAS Institute Inc, Cary, NC, USA) was used for the statistical analysis. A Fisher's exact test was used to determine the significance of $\mathrm{K}$-wire migration between the groups. A Chi-square test was used to determine differences in radiographic scoring of callus formation. Interobserver reliability was assessed using Cohen's kappa tests. Three-way analysis of variance with side and frequency as repeated measures and repair type as the nonrepeated factor was used to determine differences for elastic stiffness, viscous stiffness, and phase angle for all groups. Lastly, differences between the contralateral and repaired femoral constructs were examined using repeated-measures one-way analysis of variance. Statistical significance was taken as $P<0.05$.

\section{Results}

\section{In vivo functional (clinical) outcomes}

Postoperatively, the rats did not have any impaired gait patterns, and all were observed to stand on their hind legs. None of the rats experienced wound infection. Two rats, one with scaffold alone and one with implanted scaffold/hMSCs, were sacrificed because of K-wire migration through the skin. Partial K-wire migration was observed in seven rats. However, no statistically significant difference was found in the rate of partial $\mathrm{K}$-wire migration $(P=0.82)$ among all groups.

\section{Radiographic testing}

Radiographs were taken at 0 and 4 months postoperatively to assess radiographic healing (Figure 4). For the autograft specimens, clear osteotomy cuts are distinctly visible at time 0 , but later become undetectable at 4 months (Figure 4A). Similarly, callus formations are noted in the 4-month postoperative allograft repairs, and the location of the allograft cannot be distinctly identified from the host bone on radiograph (Figure 4B). Because the scaffold is radiolucent, a large void is seen for the scaffold femoral repairs. For both scaffold repairs without hMSCs (Figure 4C) and with hMSCs (Figure 4D), some callus formation can be seen around the host-scaffold interphase at four months.

In addition, all samples were evaluated by radiographic scoring criteria. Both scaffold groups have significantly less bridging callus than the autograft group $(P<0.05)$. There was no significant difference in the amount of bridging callus observed between the autograft and allograft groups $(P=0.59)$. Overall agreement for interobserver reliability was fair, with a kappa value of $0.37(P<0.0001)$.
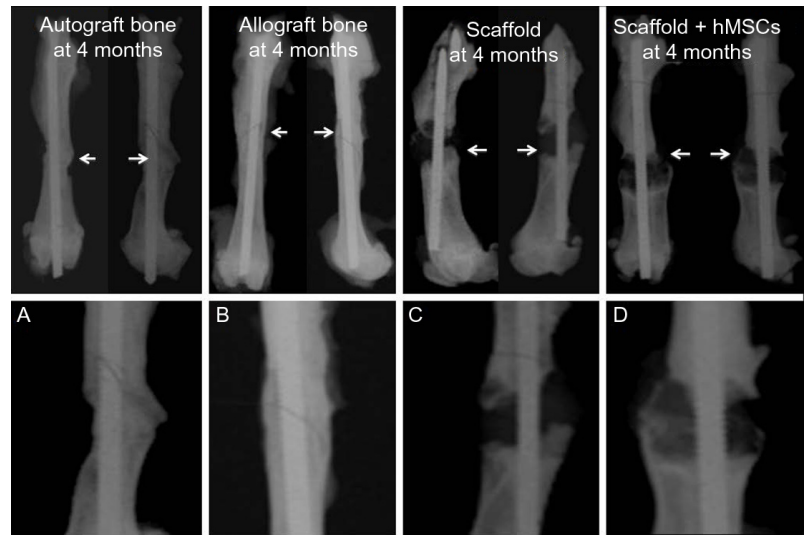

Figure 4 Radiographic healing of femoral repairs. Callus formation is noted in the 4-month postoperative autograft samples (A) and allograft samples (B). The 4-month postoperative scaffold samples with and without human mesenchymal stem cells (C and $\mathbf{D})$ also demonstrate signs of radiographic healing. 


\section{Biomechanical testing}

All femoral repairs and their contralateral controls underwent dynamic mechanical analysis at $0.5,1.0$, and $5.0 \mathrm{~Hz}$. At $5.0 \mathrm{~Hz}$, some repairs in the scaffold and allograft groups broke during testing. Because of the limited remaining sample size, the scaffold group was not included in the $5 \mathrm{~Hz}$ data analysis.

Elastic stiffness was found to be significantly higher for the autograft and allograft groups than for the scaffold and scaffold/hMSC groups $(P=0.002)$. At $0.5 \mathrm{~Hz}$ and $1.0 \mathrm{~Hz}$, both the autograft and allograft repairs had significantly higher elastic stiffness than the scaffold groups $(P=0.002)$. Using one-way analysis of variance, the allograft group, the scaffold group, and the hMSC-loaded scaffold group were found to have significantly less elastic stiffness than their contralateral control groups at both 0.5 and $1.0 \mathrm{~Hz}$ $(P<0.05$, Figure 5). Lastly, at $5.0 \mathrm{~Hz}$, the autograft repair group had significantly higher elastic stiffness than the other repair groups $(P=0.0046)$, while the allograft and scaffold/hMSC repair groups had significantly lower elastic stiffness than their contralateral control groups $(P=0.044$ and $P=0.021$ ).

Viscous stiffness was also measured for all femoral repairs. At $0.5 \mathrm{~Hz}$, viscous stiffness was not significantly different among the repair groups. However, the scaffold group had significantly lower viscous stiffness than its control group $(P=0.013)$. At $1.0 \mathrm{~Hz}$, the viscous stiffness was significantly higher in the autograft, allograft, and scaffold/hMSC repair groups than in the scaffold alone group $(P=0.008)$. The allograft and scaffold repair groups had lower viscous stiffness than their contralateral control groups ( $P=0.024$ and $P=0.009$, respectively, Figure 6).

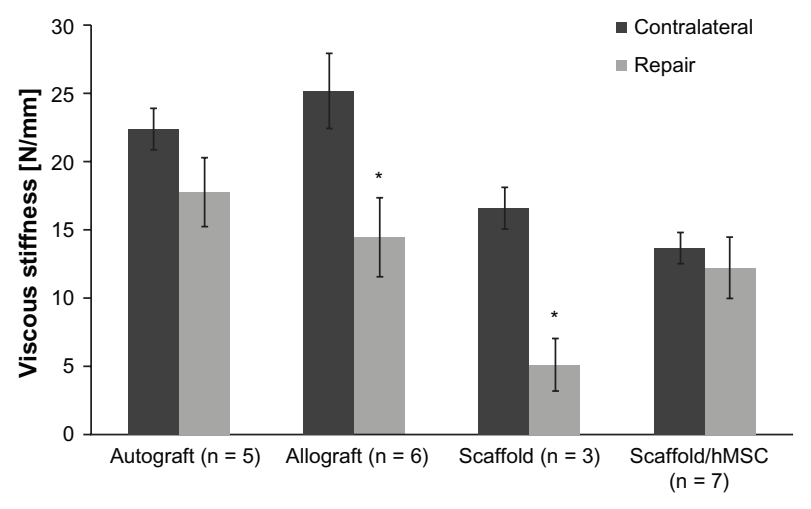

Figure 6 Viscous stiffness of femoral repairs at $1.0 \mathrm{~Hz}$.

Notes: The viscous stiffness values at $1.0 \mathrm{~Hz}$ for the autograft and scaffold/human mesenchymal stem cell repair groups are not significantly different from that of their contralateral control groups. However, the viscous stiffness is significantly less for the allograft and scaffold repairs than their contralateral controls $(* P<0.05)$.

At $5.0 \mathrm{~Hz}$, the autograft group had higher viscous stiffness than all the other repair groups $(P=0.0026)$, while none of the repair groups had significantly different viscous stiffness when compared with their contralateral controls $(P>0.069)$.

At $0.5 \mathrm{~Hz}$ and $1.0 \mathrm{~Hz}$, the scaffold repair group was the only group that had a larger phase angle than its contralateral controls $(P=0.07$ and $P=0.018$, respectively, Figure 7). At $5.0 \mathrm{~Hz}$, the autograft repair group had a significantly higher phase angle than the other repair groups $(P=0.0069)$. However, none of the groups had significantly different phase angles when compared with their contralateral control groups $(P>0.272)$. Lastly, there was no significant difference in the phase angle among any of the control groups.

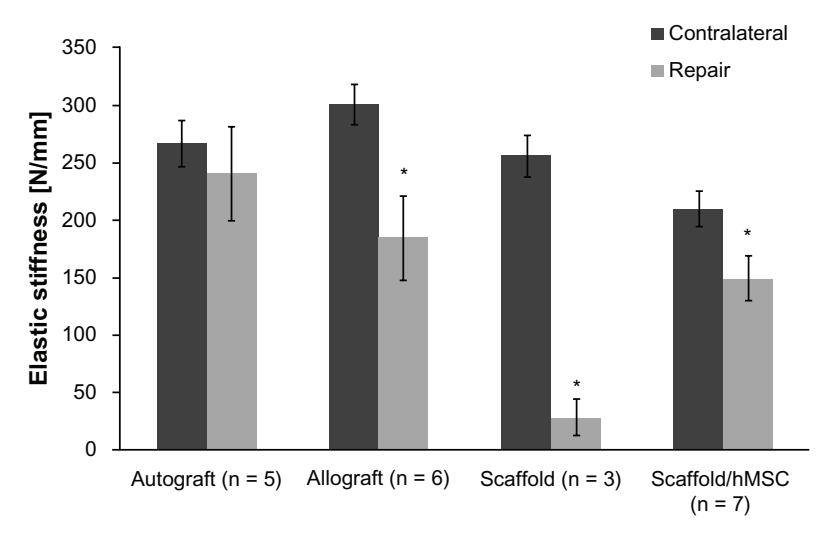

Figure 5 Elastic stiffness of femoral repairs at $1.0 \mathrm{~Hz}$.

Notes: We compared the elastic stiffness between repaired femurs and their contralateral control groups. There is no significant difference in elastic stiffness between the autograft group and its control group. However, all other repairs have significantly less elastic stiffness compared with their contralateral controls $(* P<0.05)$.

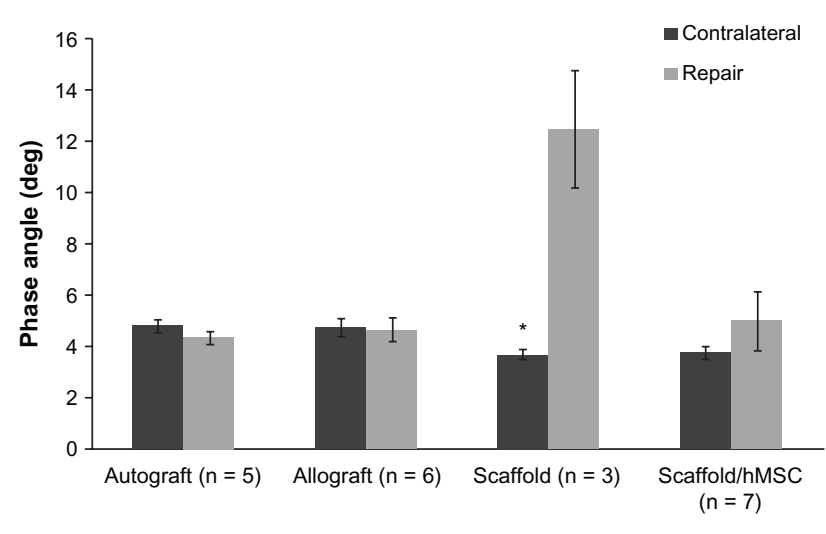

Figure 7 Phase angle of femoral repairs at $1.0 \mathrm{~Hz}$.

Notes: For the autograft, allograft, and scaffold/human mesenchymal stem cell repair groups, the phase angles at $1.0 \mathrm{~Hz}$ are not significantly different from the phase angles of their contralateral control groups. The phase angle is significantly larger for the scaffold repair group than for its control group $(P=0.018)$. 


\section{Discussion}

In this study, we sought to compare the physiologic load-bearing response of a PCL-HA scaffold with that of the current treatment standards for intercalary defect fillers, ie, autograft and allograft. There were no significant differences in K-wire migration rates among the four repair groups. Interestingly, addition of hMSCs to the PCL-HA scaffold enhanced the mechanical properties of the repair and promoted further bone formation. Furthermore, radiographic healing was a poor indicator for restoration of the mechanical properties of the femoral repairs.

For all frequencies, the scaffold alone group has significantly lower elastic stiffness, lower viscous stiffness, and higher phase angles than the other repair groups and their contralateral controls. Lower viscoelastic stiffness suggests that the scaffold repairs may have had incomplete bridging callus formation, resulting in structures that were less stiff in response to applied loads. However, addition of hMSCs onto the scaffold dramatically increased the elastic stiffness and viscous stiffness of the repair, while decreasing the phase angle. The viscous stiffness and phase angle for the scaffold/ hMSCs repairs were not significantly different from their contralateral controls. Hence, our data suggest that when hMSCs are added to the scaffold, more natural biomechanical properties are restored.

hMSCs also appear to promote bone growth. The scaffold/ hMSCs repairs showed significantly more bridging callus than the scaffold alone repairs. In a previous study where a PCL-HA scaffold was implanted into an osteoarticular defect in the rabbit proximal humerus, bone formation was noted in a scaffold group loaded with transforming growth factor- $\beta 3{ }^{8}$ Similarly, another study found that PCL-HA scaffold loaded with hMSCs improved bone formation. ${ }^{7}$ Hence, we conclude that a PCL-HA scaffold is an effective carrier for hMSCs. Moreover, hMSCs not only enhance the mechanical properties of the scaffold but also promote bone formation.

Another notable finding is that the radiographic healing observed in the femoral repair groups did not reflect restoration of the mechanical properties of the repair. There are no well established clinical criteria to evaluate bone healing. ${ }^{25}$ Although there is no consensus on the best method to determine fracture healing, the number of bridging calluses has been previously shown to have the best interobserver and intraobserver reliability when grading healing in fractures treated with an intramedullary nail. ${ }^{25,26}$ In this study, we evaluated radiographs for bridging calluses, and as expected, we noted significant healing for the autograft and allograft repairs. Interestingly, even though allograft repairs had a high grade of callus bridging radiographically, the repairs were not biomechanically stiffer than the scaffold repairs, which showed much lower radiographic healing. Similarly, the scaffold/hMSC group had a significantly lower number of calluses than the allograft repairs. However, the viscous stiffness for the scaffold/hMSC group was not significantly different from that of its contralateral controls, whereas the allograft group had significantly less viscous stiffness when compared with its controls (Figure 6). Therefore, radiographic healing in femoral repairs does not truly reflect the mechanical properties of the repair. Moreover, the interobserver reliability was only fair (kappa value $=0.37$ ). Hence, radiographic evaluation may be an inadequate means for clinical evaluation of fracture healing.

Limitations in our study include the differing radiolucency of the grafts, low sample numbers, and restricted biomechanical testing. The PCL-HA scaffold is radiolucent, which allows visual tracking of new bone formation around the host-graft interface. However, autograft and allograft repairs are radio-opaque, and we cannot distinguish them from newly formed bone using x-rays or microcomputed tomography. This difference in radiolucency limits our ability to compare radiographic healing directly between the different groups. Our study is also limited by the low sample size because of handling problems and mid-test failures. Finally, load to failure tests, such as three-point bending or torsional testing, were not examined in this study. Due to the tightly fitted K-wire through the femur, the host-graft segment junction may be disrupted if the K-wire was removed, rendering the biomechanical tests invalid. Also, we did not perform torsional testing because this method of torsion testing with intact implants with threaded K-wires has not been widely validated. Most importantly, we were interested in testing cyclic physiologic loading on the femur and chose to evaluate the repairs using dynamic mechanical analysis.

Future studies may address these limitations by using inert bone markers, a larger sample size, and nonthreaded $\mathrm{K}$-wires for fixation. Using bone markers/tags, new bone formation may be distinguished from existing radio-opaque grafts. A larger sample size would allow detailed analysis and minimize potential cumulative microdamage in the femoral structure from repeated testing. After removal of unthreaded $\mathrm{K}$-wires, future repairs could undergo failure testing. Finally, histology analysis may provide insights into bone formation around the host-graft interface.

Our study indicates that application of hMSCs on scaffolds significantly enhances the biomechanical properties of femoral repair for critical segmental defects. 
Furthermore, signs of radiographic healing may not accurately reflect the mechanical and structural properties of the repair. Further studies must be conducted to elucidate the relationship between postoperative healing time and restoration of mechanical properties. Additional means to evaluate repair healing accurately in a clinical setting remain to be explored. Lastly, comparative analysis examining the use of growth factors is needed to establish a guideline for future scaffold repairs.

\section{Acknowledgments}

This study was funded by the AO North America Kathryn Cramer Award and the Department of Orthopedic Surgery at Columbia University. We would like to thank Charles Jobin, Joshua Metzl, and Mark Vitale for performing the radiographic scoring.

\section{Disclosure}

None of the authors have any conflicts, financial or otherwise, in relation to this work.

\section{References}

1. Mankin HJ, Hornicek FJ, Raskin KA. Infection in massive bone allografts. Clin Orthop Relat Res. 2005:210-216.

2. Wheeler DL, Enneking WF. Allograft bone decreases in strength in vivo over time. Clin Orthop Relat Res. 2005:36-42.

3. Lee FY, Sinicropi SM, Lee FS, Vitale MG, Roye DP Jr, Choi IH. Treatment of congenital pseudarthrosis of the tibia with recombinant human bone morphogenetic protein-7 (rhBMP-7). A report of five cases. J Bone Joint Surg Am. 2006;88:627-633.

4. Griffin M, Iqbal SA, Bayat A. Exploring the application of mesenchymal stem cells in bone repair and regeneration. J Bone Joint Surg Br. 2011;93: 427-434.

5. Shikinami Y, Okazaki K, Saito M, et al. Bioactive and bioresorbable cellular cubic-composite scaffolds for use in bone reconstruction. $J R$ Soc Interface. 2006;3:805-821.

6. Rai B, Oest ME, Dupont KM, Ho KH, Teoh SH, Guldberg RE. Combination of platelet-rich plasma with polycaprolactone-tricalcium phosphate scaffolds for segmental bone defect repair. J Biomed Mater Res A. 2007;81:888-899.

7. Lee CH, Marion NW, Hollister S, Mao JJ. Tissue formation and vascularization in anatomically shaped human joint condyle ectopically in vivo. Tissue Eng Part A. 2009;15:3923-3930.

8. Lee CH, Cook JL, Mendelson A, Moioli EK, Yao H, Mao JJ. Regeneration of the articular surface of the rabbit synovial joint by cell homing: a proof of concept study. Lancet. 2010;376:440-448.
9. Feighan JE, Davy D, Prewett AB, Stevenson S. Induction of bone by a demineralized bone matrix gel: a study in a rat femoral defect model. J Orthop Res. 1995;13:881-891.

10. Bruder SP, Kurth AA, Shea M, Hayes WC, Jaiswal N, Kadiyala S. Bone regeneration by implantation of purified, culture-expanded human mesenchymal stem cells. J Orthop Res. 1998;16:155-162.

11. Isobe M, Yamazaki Y, Mori M, Amagasa T. Bone regeneration produced in rat femur defects by polymer capsules containing recombinant human bone morphogenetic protein-2. J Oral Maxillofac Surg. 1999;57: 695-698.

12. Lee FY, Hazan EJ, Gebhardt MC, Mankin HJ. Experimental model for allograft incorporation and allograft fracture repair. J Orthop Res. 2000;18:303-306.

13. Lee FY, Storer S, Hazan EJ, Gebhardt MC, Mankin HJ. Repair of bone allograft fracture using bone morphogenetic protein-2. Clin Orthop Relat Res. 2002:119-126.

14. Seo SW, Cho SK, Storer SK, Lee FY. Zoledronate reduces unwanted bone resorption in intercalary bone allografts. Int Orthop. 2010;34: 599-603.

15. Conflitti J, Graves M, Russell G, Tucci M, Benghuzzi H. Plating of rat femoral shaft osteotomies: report of a technique and preliminary results. Biomed Sci Instrum. 2003;39:278-283.

16. Kokubu T, Hak DJ, Hazelwood SJ, Reddi AH. Development of an atrophic nonunion model and comparison to a closed healing fracture in rat femur. J Orthop Res. 2003;21:503-510.

17. Schoen M, Rotter R, Schattner S, et al. Introduction of a new interlocked intramedullary nailing device for stabilization of critically sized femoral defects in the rat: a combined biomechanical and animal experimental study. J Orthop Res. 2008;26:184-189.

18. Lynch JA, Silva MJ. In vivo static creep loading of the rat forelimb reduces ulnar structural properties at time-zero and induces damagedependent woven bone formation. Bone. 2008;42:942-949.

19. Bowman SM, Guo XE, Cheng DW, et al. Creep contributes to the fatigue behavior of bovine trabecular bone. J Biomech Eng. 1998;120: 647-654.

20. Kim DG, Miller MA, Mann KA. A fatigue damage model for cementbone interface. J Biomech. 2004;37:1505-1512.

21. Yeni YN, Kim DG, Dong XN, Turner AS, Les CM, Fyhrie DP. Do sacrificial bonds affect the viscoelastic and fracture properties of bone? Clin Orthop Relat Res. 2006;443:101-108.

22. Yamashita J, Furman BR, Rawls HR, Wang X, Agrawal CM. The use of dynamic mechanical analysis to assess the viscoelastic properties of human cortical bone. J Biomed Mater Res. 2001;58:47-53.

23. Cullen DM, Smith RT, Akhter MP. Time course for bone formation with long-term external mechanical loading. J Appl Physiol. 2000;88: 1943-1948.

24. Menard KP. Dynamic mechanical analysis: a practical introduction. Boca Raton, FL: CRC Press; 1999.

25. Corrales LA, Morshed S, Bhandari M, Miclau T 3rd. Variability in the assessment of fracture-healing in orthopaedic trauma studies. $J$ Bone Joint Surg Am. 2008;90:1862-1868.

26. Whelan DB, Bhandari M, McKee MD, et al. Interobserver and intraobserver variation in the assessment of the healing of tibial fractures after intramedullary fixation. J Bone Joint Surg Br. 2002;84:15-18.
International Journal of Nanomedicine

\section{Publish your work in this journal}

The International Journal of Nanomedicine is an international, peerreviewed journal focusing on the application of nanotechnology in diagnostics, therapeutics, and drug delivery systems throughou the biomedical field. This journal is indexed on PubMed Central,

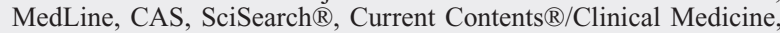

\section{Dovepress}

Journal Citation Reports/Science Edition, EMBase, Scopus and the Elsevier Bibliographic databases. The manuscript management system is completely online and includes a very quick and fair peer-review system, which is all easy to use. Visit http://www.dovepress.com/ testimonials.php to read real quotes from published authors. 